\title{
ON THE FIELD OF VALUES OF A MATRIX
}

\section{LOUIS BRICKMAN}

1. Introduction. The idea of the field of values of a complex $n \times n$ matrix $C$ was introduced by O. Toeplitz [3]. It is the set of complex numbers defined by

$$
W(C)=\left\{V^{T} C \bar{V} \mid V^{T} \bar{V}=1\right\}, \quad(V \text { an } n \times 1 \text { vector }) .
$$

$W$ is clearly a compact and connected set. Toeplitz showed in [3] that $W$ has a convex outer boundary, and a short time later $\mathrm{F}$. Hausdorff [1] proved that $W$ itself is convex. Since then several investigations have been made concerning the geometry of $W$. An example of a recent one is the dissertation of R. Kippenhahn [2] in which $W$ is described as the convex hull of a certain algebraic curve of degree $n$ obtainable from $C$.

Writing

$$
C=A+i B,
$$

the unique Hermitian decomposition of $C$, we can exhibit $W$ in the real coordinate form

$$
W(A, B)=\left\{\left(V^{T} A \bar{V}, V^{T} B \bar{V}\right) \mid V^{T} \bar{V}=1\right\} .
$$

This representation suggests a generalization to more than two Hermitian forms, but it is easy to show by example that convexity does not survive this extension. It is known, however, (see $[1 ; 3]$ ) that for three forms the boundary of $W$ is still convex.

We shall be concerned with the real analog of (1.3), i.e., the set

$$
\text { (1.4) } R(A, B)=\left\{\left(V^{T} A V, V^{T} B V\right) \mid V^{T} V=1\right\} ; \quad A, B, V \text { real. }
$$

With the understanding (henceforth in force) that $A$ and $B$ are real symmetric matrices, we obviously have

$$
R(A, B) \subset W(A, B) .
$$

In $\S 2$ we shall show that for $n \geqq 3$ we actually have

$$
R(A, B)=W(A, B) \text {. }
$$

In other words the nonreal vectors in (1.3) only duplicate the contributions of the real ones. This phenomenon is familiar in the case of one Hermitian form based on a real matrix, but it does not occur in the general case of three or more forms. (See Remark 4.) Indeed,

Received by the editors March 30, 1960. 
the proof of Theorem 2.2 shows that it will occur if and only if $R$ is convex. Hence this convexity is the first result established.

In $\S 3$ the sets obtained by removing the conditions $V^{T} \bar{V}=1$ and $V^{T} V=1$ are described. Using the results of $\$ 2$ we find that

$$
\hat{W}(A, B)=\left\{\left(V^{T} A \bar{V}, V^{T} B \bar{V}\right) \mid V \text { complex }\right\}
$$

and

$$
\hat{R}(A, B)=\left\{\left(V^{T} A V, V^{T} B V\right) \mid V \text { real }\right\}
$$

are convex cones with

$$
\hat{W}(A, B)=\hat{R}(A, B)
$$

for every $n$. Finally it is observed that the set

$$
\hat{W}(H, K, L)=\left\{\left(V^{T} H \bar{V}, V^{T} K \bar{V}, V^{T} L \bar{V}\right) \mid V \text { complex }\right\}
$$

is a convex cone in three-space. ( $H, K, L$, any Hermitian matrices.)

2. Proof of (1.5). We can easily establish, exactly as Toeplitz did for (1.1), that $R$ has a convex outer boundary for all $n$. Let us rather recall the method of Hausdorff. He showed that the inverse image under (1.1) of every line

$$
a x+b y+c=0
$$

of the $x y$ plane is a connected subset of the unitary unit sphere. From this it follows that every line intersects $W$ in a connected set, whence $W$ is convex. This method has to be modified for the real case (1.4), however, as the following example shows. Taking $n=3$ let

$$
\begin{aligned}
& x=V^{T} A V=v_{1}^{2}-v_{2}^{2}-v_{3}^{2} \\
& y=V^{T} B V \text { (arbitrary) } \quad\left(v_{1}^{2}+v_{2}^{2}+v_{3}^{2}=1\right) .
\end{aligned}
$$

The inverse image under (2.1) of the line $x=1$ consists of the two points $(1,0,0)$ and $(-1,0,0)$, a disconnected set. In spite of this we can still conclude that $x=1$ intersects $R$ in a connected set; viz., the above set consists of two connected components which are symmetric about $(0,0,0)$. Hence they both have the same (connected) image under (2.1), and the conclusion follows. This idea is the key to the following theorem.

Theorem 2.1. If $n \geqq 3, R(A, B)$ is convex.

Proof. It is convenient to work with $n-1$ dimensional projective space $P_{n-1}$. Thus $R$ is the image of $P_{n-1}$ under the mapping 


$$
\begin{aligned}
& x=V^{T} A V / V^{T} V, \\
& y=V^{T} B V / V^{T} V .
\end{aligned}
$$

We shall prove that the inverse image under (2.2) of every line

$$
a x+b y+c=0
$$

is connected in $P_{n-1}$. Such a set is defined by

$$
a V^{T} A V / V^{T} V+b V^{T} B V / V^{T} V+c=0
$$

or

$$
V^{T}(a A+b B+c I) V=0 .
$$

Hence the theorem reduces to the assertion that a real projective hyperconic is a connected set. This can be shown by induction. If $n=3$, the smallest value being considered, $(2.3)$ is an ordinary projective conic $Q_{2}$, which is connected. (The nondegenerate conics are all topological circles, and the degenerate cases of (2.3) are the null set, a point, a line, a pair of lines, all of $P_{n-1}$.) Suppose that all hyperconics $Q_{n-1}$ in $P_{n-1}$ are connected, $n \geqq 3$, and let $Q_{n}$ be a hyperconic in $P_{n}$. Through two arbitrary points of $Q_{n}$ construct a hyperplane $P_{n-1}$. Then $Q_{n} \cap P_{n-1}$ is a hyperconic $Q_{n-1}$, which is connected. (That $Q_{n} \cap P_{n-1}$ is a hyperconic follows at once from the fact that the equation of $P_{n-1}$ can be taken to be $v_{n}=0$ without loss of generality.) Thus any two points of $Q_{n}$ belong to a connected subset of $Q_{n}$. The theorem now follows.

REMARK 1. Although the unitary analog of Theorem 2.1 holds for any $n$, Theorem 2.1 fails for $n=2$. Let

$$
\begin{aligned}
& x=v_{1}^{2}-v_{2}^{2}, \\
& y=2 v_{1} v_{2} .
\end{aligned}
$$

Then $R$ is the unit circle $x^{2}+y^{2}=1$. More generally, it can be shown that for $n=2, R$ is either an ellipse, a circle, a line segment, or a point.

REMARK 2. The theorem also breaks down if the number of quadratic forms is increased. For example let $n=3$ and consider the three forms

$$
\begin{aligned}
& x=v_{1}^{2}-v_{2}^{2}, \\
& y=2 v_{1} v_{2}, \\
& z=v_{3}^{2} .
\end{aligned}
$$


The intersection of $R$ and the supporting plane $z=0$ is again the circle $x^{2}+y^{2}=1$. Hence $R$ does not even have a convex boundary.

Theorem 2.2. If $n \geqq 3, R(A, B)=W(A, B)$.

Proof. We need only show that $W \subset R$. Let

$$
(x, y) \in W(A, B) \text {. }
$$

Then for some complex unit vector $V$

$$
\begin{aligned}
& x=V^{T} A \bar{V}, \\
& y=V^{T} B \bar{V} .
\end{aligned}
$$

Let

$$
V=V_{1}+i V_{2}
$$

Then

$$
x=\left(V_{1}^{T}+i V_{2}^{T}\right) A\left(V_{1}-i V_{2}\right)=V_{1}^{T} A V_{1}+V_{2}^{T} A V_{2},
$$

and similarly

$$
y=V_{1}^{T} B V_{1}+V_{2}^{T} B V_{2} .
$$

Since $V_{1}^{T} V_{1}+V_{2}^{T} V_{2}=1$, it is clear that $(x, y) \in R$ if either $V_{1}=0$ or $V_{2}=0$. In all other cases we can write

$$
\begin{aligned}
& x=V_{1}^{T} V_{1}\left(U_{1}^{T} A U_{1}\right)+V_{2}^{T} V_{2}\left(U_{2}^{T} A U_{2}\right), \\
& y=V_{1}^{T} V_{1}\left(U_{1}^{T} B U_{1}\right)+V_{2}^{T} V_{2}\left(U_{2}^{T} B U_{2}\right),
\end{aligned}
$$

where $U_{1}$ and $U_{2}$ are the unit vectors

$$
\begin{aligned}
& U_{1}=\left(V_{1}^{T} V_{1}\right)^{-1 / 2} V_{1}, \\
& U_{2}=\left(V_{2}^{T} V_{2}\right)^{-1 / 2} V_{2} .
\end{aligned}
$$

Hence $(x, y)$ is a convex combination of two points of $R$, and therefore belongs to $R$.

REMARK 3. Although we can have $R \neq W$ for $n=2$, it is now clear that $W$ is the convex hull of $R$ in any case.

REMARK 4. The generalization of Theorem 2.2 to more than two forms is false. The quadratic forms of Remark 2 and the corresponding Hermitian forms provide a simple counterexample.

The following result is a generalization of Theorem 2.2 . 
Corollary. Let $C$ be any complex $n \times n$ matrix, $n \geqq 3$, and let

$$
R(C)=\left\{V^{T} C V \mid V \text { real, } V^{T} V=1\right\} .
$$

Then

$$
R(C)=W\left(\frac{1}{2} C+\frac{1}{2} C^{T}\right)
$$

Proof. If

$$
C=H+i K
$$

then

$$
\frac{1}{2} C+\frac{1}{2} C^{T}=\operatorname{Re} H+i \operatorname{Re} K .
$$

Hence

$$
\begin{aligned}
R(C) & =R(H, K)=R(\operatorname{Re} H, \operatorname{Re} K)=W(\operatorname{Re} H, \operatorname{Re} K) \\
& =W\left(\frac{1}{2} C+\frac{1}{2} C^{T}\right)
\end{aligned}
$$

3. Arbitrary vectors. We wish now to discuss the removal of the conditions $V^{T} \bar{V}=1$ and $V^{T} V=1$. For example in place of (1.1) we can define

$$
\hat{W}(C)=\left\{V^{T} C \bar{V} \mid V \text { complex }\right\}
$$

Then

$$
\hat{W}(C)=\{r z \mid r \geqq 0, z \in W(C)\} .
$$

This, together with the convexity of $W$, implies that $\hat{W}$ is a convex cone. Thus if $r_{1} z_{1}$ and $r_{2} z_{2}$ belong to $\hat{W}$, and if $a_{1}$ and $a_{2}$ are any nonnegative numbers, then

$$
\begin{aligned}
a_{1} r_{1} z_{1}+a_{2} r_{2} z_{2}= & \left(a_{1} r_{1}+a_{2} r_{2}\right)\left(a_{1} r_{1}\left(a_{1} r_{1}+a_{2} r_{2}\right)^{-1} z_{1}\right. \\
& \left.+a_{2} r_{2}\left(a_{1} r_{1}+a_{2} r_{2}\right)^{-1} z_{2}\right) \in \hat{W}(C) .
\end{aligned}
$$

(This holds trivially if $a_{1} r_{1}+a_{2} r_{2}=0$.) Similar remarks apply to $\hat{W}(A, B)$ and, if $n \geqq 3$, to $\hat{R}(A, B)$, defined in (1.6) and (1.7) respectively. Hence Theorem 2.2 implies that $\hat{R}$ and $\hat{W}$ are equal convex cones. This conclusion holds even for $n=2$. To see this let

$$
(0,0) \neq(x, y) \in W(A, B), \quad(r x, r y) \in \hat{W}(A, B) .
$$

Since $W$ is compact, there is a positive number $s$ such that $(s x, s y)$ 
belongs to the boundary of $W$. But the boundary of $W$ is precisely $R$. (See Remarks 1 and 3.) Therefore

$$
(s x, s y) \in R(A, B),
$$

and

$$
(r x, r y)=(r / s s x, r / s s y) \in \hat{R}(A, B) .
$$

Thus

$$
\hat{W}(A, B) \subset \hat{R}(A, B),
$$

and since the opposite containment is obviously correct, the above assertion is proved.

Finally, let $H, K, L$, be Hermitian matrices. Then

$$
W(H, K, L)=\left\{\left(V^{T} H \bar{V}, V^{T} K \bar{V}, V^{T} L \bar{V}\right) \mid V^{T} \bar{V}=1\right\}
$$

has a convex boundary. By reasoning similar to that just used, we conclude that $\hat{W}(H, K, L)$, defined in (1.9), is a convex cone. Thus we have the following theorem.

TheOREM 3.1. Let $\hat{W}(A, B), \hat{R}(A, B), \hat{W}(C)$, and $\hat{W}(H, K, L)$ be the sets defined in (1.6), (1.7), (3.1), and (1.9) respectively. Then each of these sets is a convex cone. Moreover,

$$
\hat{W}(A, B)=\hat{R}(A, B)
$$

holds for every $n$.

Acknowledgment. The author wishes to thank Dr. Harold Levine for discussions on portions of this material.

\section{REFERENCES}

1. Felix Hausdorff, Der Wertvorrat einer Bilinearform, Math. Z. vol. 3 (1919) pp. 314-316.

2.Rudolf Kippenhahn, Über den Wertevorrat einer Matrix, Math. Nachr. vol. 6 (1951) pp. 193-228.

3. Otto Toeplitz, Das algebraische Analogon zu einem Satze von Fejêr, Math. Z. vol. 2 (1918) pp. 187-197.

YALE UNIVERSITY 